\title{
Erratum to: Chronic and Invasive Fungal Infections in a Family with CARD9 Deficiency
}

\author{
Ana Karina Alves de Medeiros ${ }^{1}$ Evelyn Lodewick ${ }^{1}$ - Delfien J. A. Bogaert ${ }^{2,3,4}$. \\ Filomeen Haerynck $^{2,3}$ - Sabine Van daele ${ }^{2}$. Bart Lambrecht ${ }^{3,4,5}$ - Sara Bosma $^{1}$. \\ Laure Vanderdonckt $^{1}$ • Olivier Lortholary ${ }^{6,7}$ - Mélanie Migaud ${ }^{6,8}$. \\ Jean-Laurent Casanova ${ }^{6,8,9,10,11}$ • Anne Puel ${ }^{6,8}$ - Fanny Lanternier ${ }^{6,7,8}$ • Jo Lambert ${ }^{1}$. \\ Lieve Brochez ${ }^{1}$. Melissa Dullaers ${ }^{3,4}$
}

Published online: 26 April 2016

(C) Springer Science+Business Media New York 2016

Erratum to: J Clin Immunol (2016)

DOI 10.1007/s10875-016-0255-8

The original version of this article did not include the full list of affiliations for author Delfien J. A. Bogaert. The correct list of affiliations includes:

2 Department of Pediatric Pulmonology and Immunology, Centre for Primary Immune deficiencies, Ghent University Hospital, Ghent, Belgium

3 Clinical Immunology Research Laboratory, Department of Respiratory Medicine, Ghent University Hospital, Ghent, Belgium

4 Laboratory for Mucosal Immunology, VIB Inflammation Research Center, Ghent, Belgium

The online version of the original article can be found at http://dx.doi.org/ 10.1007/s10875-016-0255-8.

Ana Karina Alves de Medeiros

ana.alvesdemedeiros@uzgent.be

1 Department of Dermatology, Ghent University Hospital, Medical Research Building ground floor, De Pintelaan 185, 9000 Ghent, Belgium

2 Department of Pediatric Pulmonology and Immunology, Centre for Primary Immune deficiencies, Ghent University Hospital, Ghent, Belgium

3 Clinical Immunology Research Laboratory, Department of Respiratory Medicine, Ghent University Hospital, Ghent, Belgium

4 Laboratory for Mucosal Immunology, VIB Inflammation Research Center, Ghent, Belgium
5 Department of Respiratory Medicine, Ghent University Hospital, Ghent, Belgium

6 Paris Descartes University, Imagine Institute, Paris, France

7 Infectious Diseases Unit, Necker-Enfants Malades Hospital, AP-HP Paris, Paris, France

8 Laboratory of Human Genetics of Infectious Diseases, Necker Branch; INSERM UMR1163, Necker Medical School, Paris, France

9 St Giles Laboratory of Human Genetics of Infectious Diseases, Rockefeller Branch, Rockefeller University, New York, NY, USA

10 Pediatric Hematology-Immunology-Rheumatology Unit, AP-HP, Necker Enfants-Malades Hospital, Paris, France

11 Howard Hughes Medical Institute, New York, NY, USA 